\title{
MRI appearances of the facial nerve in an HIV infected patient with acute facial nerve palsy
}
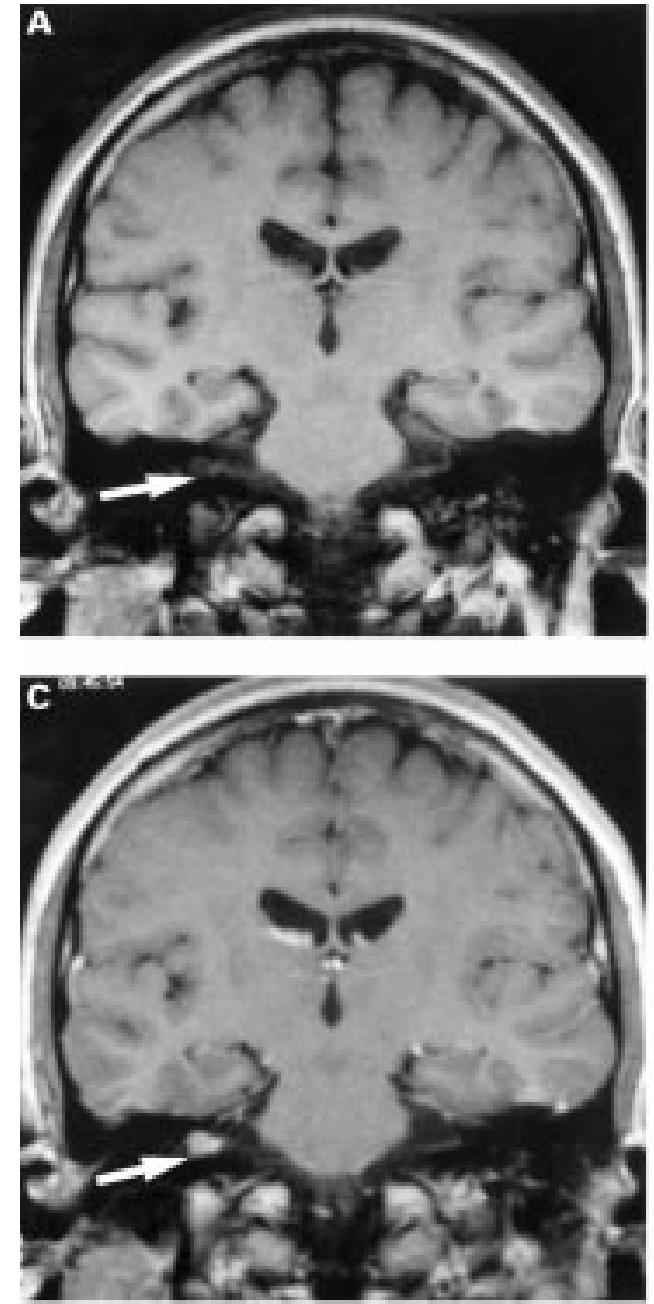

A 27 year old white HIV positive homosexual man presented with a 6 day history of right mandibular and posterior auricular pain progressing to right facial weakness on day 7 . Examination showed a right lower motor neuron facial palsy. On MRI performed on day 7, coronal (fig A) and axial (fig B) T1 weighted unenhanced images showed swelling (arrow) and increased signal (curved arrow) of the intracanalicular segment of the right facial nerve. Corresponding images post-Gd-DTPA (figs C, D) showed enhancement of the right intracanalicular segment (arrow) and the geniculate ganglion (curved open arrow). In view of the well preserved CD4+ lymphocyte count of 460 $\times 10^{6} / 1$ (normal: $\left.350-2200 \times 10^{6} / 1\right)$, lumbar puncture was not performed as CSF leucocytosis would not have been an indication to
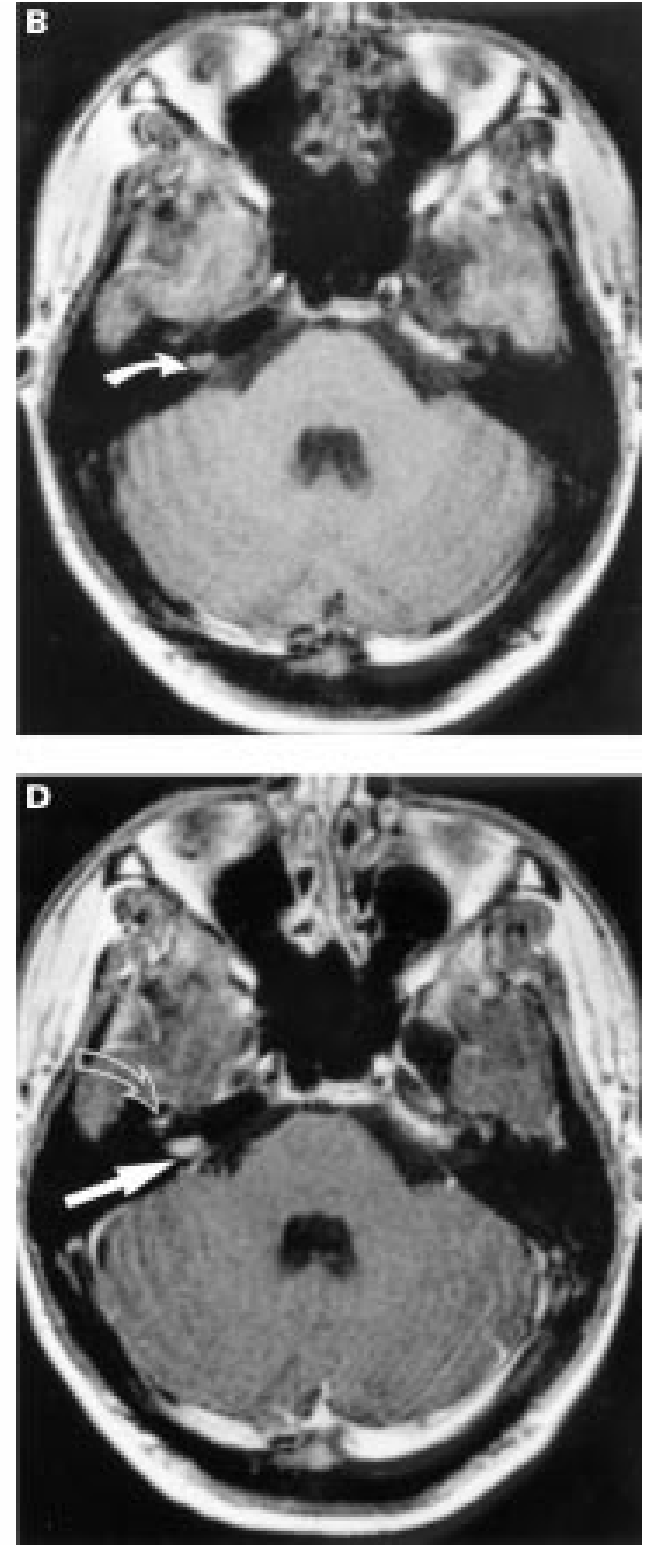

start antiretroviral medication. The patient wastreated with $60 \mathrm{mg}$ oral prednisolone daily for 10 days. Follow up MRI on day 76 (the facial palsy having completely resolved), showed reduction in swelling and enhancement of the affected facial nerve.

Facial nerve palsy is part of the clinical range of disease resulting from HIV infection and is more common in asymptomatic patients than in those with more advanced disease. ${ }^{1}$ The enhancement pattern of the affected facial 
nerve we describe is similar to that described in Bell's palsy, ${ }^{2}$ a condition in which the neurotropic Herpes simplex virus type 1 is probably the major aetiological agent ${ }^{3}$; HIV is also neurotropic and virus localisation in the facial nerve or the geniculate ganglion may cause mast cell degranulation, with release of vasoactive substances altering the permeability of the blood-peripheral nerve barrier resulting in intraneural oedema and fibre swelling. ${ }^{2}$ The end result of these interactions may be seen on MRI as swelling and pathological enhancement of the affected facial nerve.

K A MISZKIEL MRI Unit, Imaging Department at UCL Hospitals (NHS) Trust, London, UK
R F MILLER

Division of Pathology and Infectious Diseases,

UCL Medical School, Camden and Islington Community Health Services (NHS) Trust, The Middlesex Hospital, Mortimer Street, London, UK

Correspondence to: Dr Katherine Anne Miszkiel, Lysholm Department of Radiology, The National Hospital for Neurology and Neurosurgery, Queen Square, London WC1 3BG, UK. Telephone 0044171837 3611; email KAMiszkiel@aol.com 1 Belec L, Georges AJ, Vuillecard E, et al. Peripheral facial paralysis indicating HIV infection. Lancet 1988;ii:1421-2.
Sattoretti-Schefer S, Wichman W, Valavanis A. Idiopathic, Sattoretti-Schefer S, Wichman W, Valavanis A. Idiopathic,
herpetic, and HIV-associated facial nerve palsies; abnormal MR enhancement patterns. AfNR Am $\mathcal{F}$ Neuroradiol 1994; 15:479-85.

3 Murakami S, Mizobuchi M, Nakashiro Y, et al. Bell palsy and Herpes simplex virus: identification of viral DNA in endoneurial fluid and muscle. Ann Intern Med 1996;124: 27-30.

\section{NEUROLOGICAL PICTURE}

\section{Optic nerve sheath meningioma}

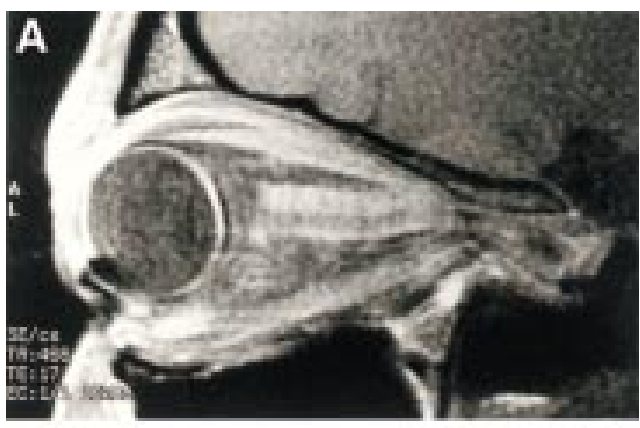

$\mathbf{B}$
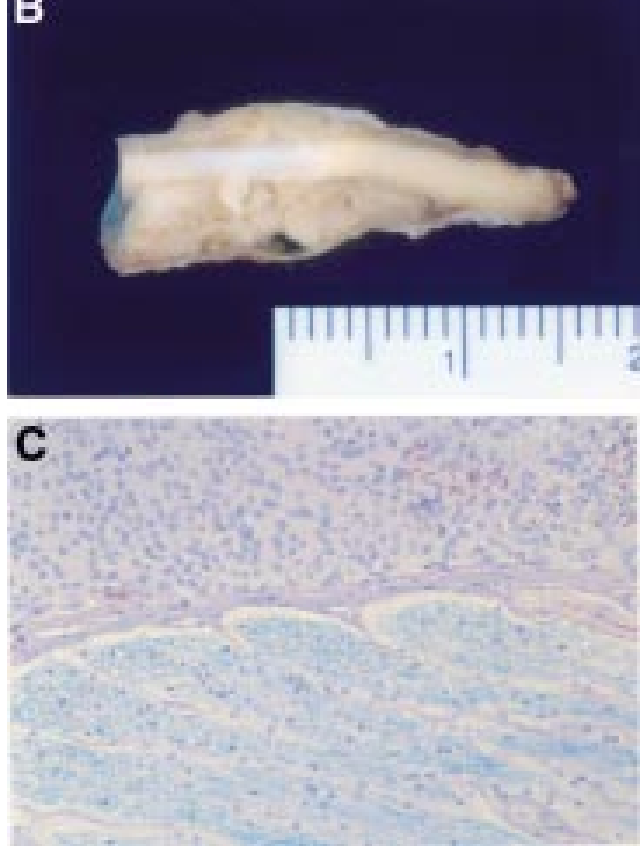

A 29 year old woman with an unremarkable medical history presented with painless progressive visual failure in the left eye. On examination, the left eye was proptotic, with a visual acuity of 20/70, and a central scotoma. There was a left relative afferent pupillary defect, and mild papilloedema involving the left optic disc. The rest of the neurological examination was normal.

A sagittal T1 weighted gadolinium enhanced image showed a normal sized left optic nerve, concentrically surrounded by a homogeneously enhancing mass extending from the globe to the optic foramen (figure A).

At surgery, in keeping with the recommended principles of operative management for such lesions, the left optic nerve was sacrificed, and gross total resection of the tumour achieved, with clear margins. A longitudinal section through the operative specimen is shown (figure $\mathrm{B}$, scale in $\mathrm{cm}$ ).

Histopathological examination confirmed the clinical diagnosis of an optic nerve sheath meningioma (figure $\mathrm{C}$, haematoxylin-eosin and luxol fast blue).

I FAYAZ

Division of Neurosurgery, St Michael's Hospital, Toronto, Ontario, Canada

F GENTILI

Division of Neurosurgery, The Toronto Hospital, Toronto, Ontario, Canada

I R MACKENZIE

Department of Pathology, Vancouver General Hospital, Vancouver, British Columbia, Canada

Correspondence to: Dr I Fayaz, Division of Neurosurgery, St Michael's Hospital, 38 Shuter Street, T oronto, Ontario M5B 1A6, Canada. Telephone 0014168645431. 


\section{Ictal PET in temporal lobe epilepsy}

A 55 year old woman with medically intractable epileptic seizure since the age of 11 years was admitted for presurgical evaluation and intensive monitoring with closed circuit television videotape using surface scalp and sphenoidal electrodes. Physical examination showed a smaller first toe, smaller thumb size, and smaller calf muscle on the left side suggesting possible right cerebral hemiatrophy. Computed tomography, 2-fluorodeoxyglucose PET imaging, and MRI of the brain in the interictal state were normal. Interictal EEG showed frequent medial and lateral anterotemporal discharges bilaterally, more frequent on the right. Video EEG telemetry recording with intracranial subdural strip electrodes, performed in 1986, could not lateralise the origin of seizures because 22 seizures clustered and appeared in succession lateralising to the right medial temporal lobe in 13 attacks and to the left medial temporal lobe in eight seizures. Complex partial status epilepticus was present at the time of isotope tracer (2-fluoro-2deoxyglucose) injection and an area of intense hypermetabolism was present over the right medial temporal lobe.

The figure shows two consecutive axial (upper left and right) and sagittal (lower left and right) images of 2-fluoro-2-deoxyglucose cerebral PET, at the level of the right medial temporal lobe. These show an intense hypermetabolism area in the right mesial temporal region.

C Y FONG

Division of Neurology, University Department of Medicine, Queen Mary Hospital, Hong Kong, China

A V DELGADO-ESCUETA

California Comprehensive Epilepsy Program and Department of Neurology, UCLA, WLA DVA Medical Centre, Los Angeles, CA, USA

Correspondence to: Dr C Y Fong, Division of Neurology, University Department of Medicine, Queen Mary Hospital, Pokfulam Road, Hong Kong, China.
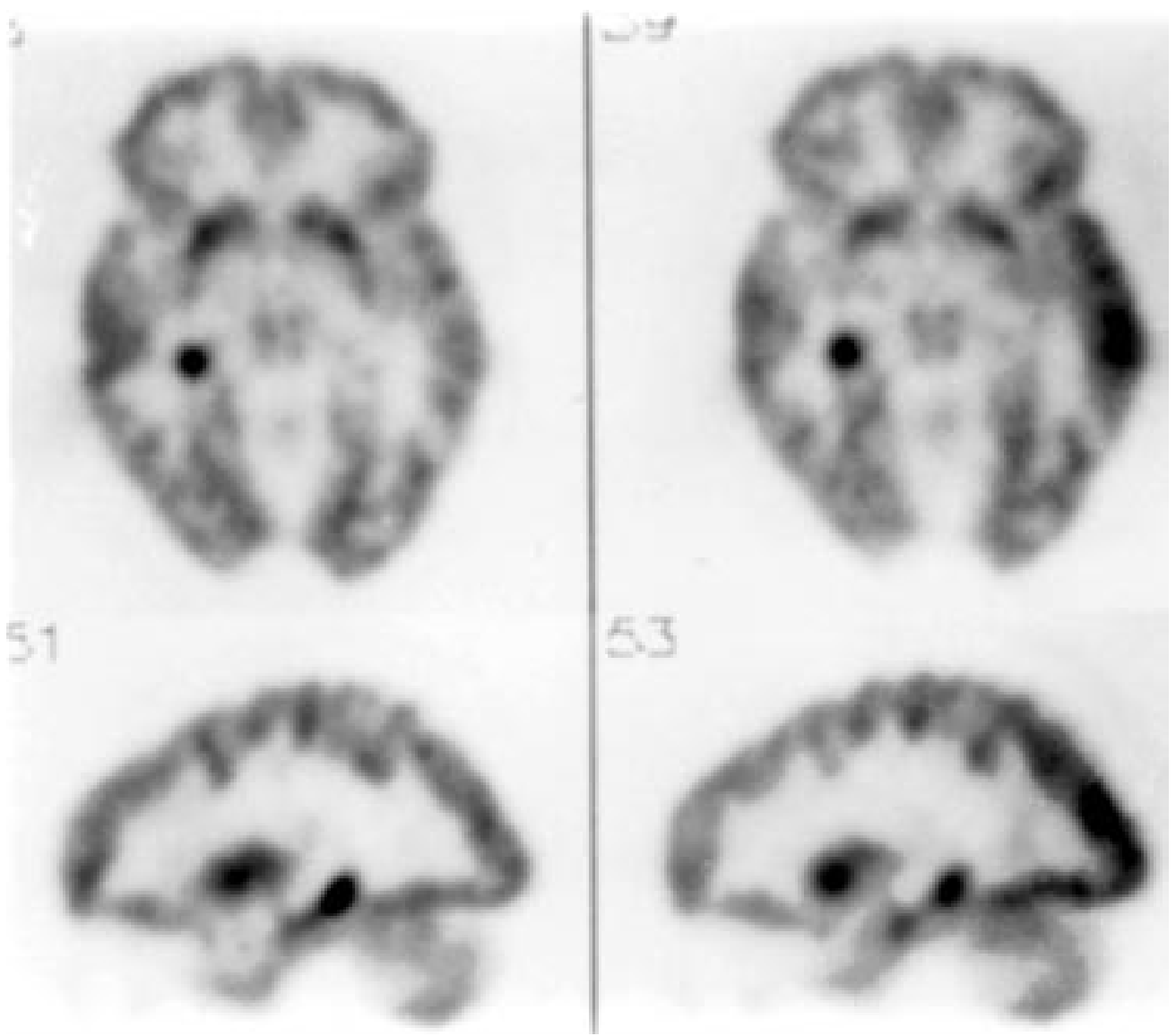